\title{
Information-disturbance tradeoff in quantum measurement on the uniform ensemble
}

\author{
Howard Barnum \\ Dept. of Computer Science, University of Bristol, Merchant Venturers Building, Woodland Rd. \\ Bristol BS8 1UB, UK.*
}

(Aug. 31, 2000)

\begin{abstract}
I consider the tradeoff between the information gained about an initially unknown quantum state, and the disturbance caused to that state by the measurement process. I show that for any distribution of initial states, the information-disturbance frontier is convex, and disturbance is nondecreasing with information gain. I consider the most general model of quantum measurements, and all post-measurement dynamics compatible with a given measurement. For the uniform initial distribution over states, I show that an optimal information-disturbance combination may always be achieved by a measurement procedure which satisfies a generalization of the projection postulate, the "square-root dynamics." I use this to show that the information-disturbance frontier for the uniform ensemble may be achieved with "isotropic" (unitarily covariant) dynamics. This results in a significant simplification of the optimization problem for calculating the tradeoff in this case, giving hope for a closed-form solution. I also show that the discrete ensembles uniform on the $d(d+1)$ vectors of a certain set of $d+1$ "mutually unbiased" or conjugate bases in $d$ dimensions form spherical 2-designs in $C P_{d-1}$ when $d$ is a power of an odd prime. This implies that many of the results of the paper apply also to these discrete ensembles.
\end{abstract}

\section{INTRODUCTION}

In this paper, I consider one of the salient ways in which quantum information differs from classical information In classical information theory, we may in principle determine the state of a system arbitrarily accurately with arbitrarily little disturbance to that state. By contrast, in quantum mechanics any measurement which allows one to obtain information

*Present address: CCS-3 (Modelling Algorithms, and Informatics), Mail Stop B256, Los Alamos National Laboratories, Los Alamos, NM 87545; barnum@lanl.gov. 
about the state of a quantum system must, on average, disturb that state, except in special cases. The special cases are when the possible states of the system are known in advance to lie in one or the other of two or more orthogonal subspaces - then the information about which of the orthogonal subspaces the state lies in can be extracted without disturbance. This fact underlies some important applications of quantum mechanics in information processing, notably quantum key distribution [1] [2] and other forms of quantum cryptography, as well as some applications to algorithms, such as the proof that PSPACE has constant-round quantum interactive proof systems [3]. The goal of this paper is to quantify the tradeoff between information gained and disturbance to the system, and derive general features of that tradeoff.

In introductory presentations of quantum theory, it is often stated that when a quantum system is measured and a result uniquely associated with a particular eigenvector of the measured observable is obtained, the system state "collapses" to that eigenvector. This is usually known as "the projection postulate," and attributed to von Neumann [4]. It clearly represents a disturbance to the system's state, unless the system is already in an eigenstate of the measured observable. A generalization of the projection postulate to observables with degenerate eigenspaces is known as "Lüders' rule;" it is slightly different from von Neumann's proposed post-measurement dynamics for that situation. Lüders' rule says that upon a measurement yielding result $b$ corresponding to a projector $\Pi_{b}$ (onto a degenerate eigenspace of the observable) an initial density operator $\rho$ evolves to

$$
\hat{\rho}_{b}^{\prime}:=\frac{\Pi_{b} \rho \Pi_{b}}{p_{b}}
$$

where $p_{b}=\operatorname{Tr} \rho \Pi_{b}$ is the probability of obtaining result $b$ [5], [6]. Hence the aftermeasurement unconditional density operator becomes $\rho^{\prime}:=\sum_{b} \Pi_{b} \rho \Pi_{b}$. But in fact this postulate describes only one of the many possible ways in which a physical process of measurement may affect a system. I will call measurements in which the effect on the system is described by the Lüders' rule form of the projection postulate projective measurements. Von Neumann's proposal, that the post-measurement density matrix conditional on observing the $b$-th outcome becomes $\Pi_{b} / \operatorname{tr} \Pi_{b}$, is another potential post-measurement dynamics which is consistent with quantum theory. (Lüders' rule, however, is a more appropriate candidate for a "generalized projection postulate," since it describes the conditional dynamics of measurement via a projection.) In the next section, I will review a more general description both of measurements (as Positive Operator Valued Measures (POVM's)) and of their effects on the system (as a collection of trace-decreasing completely positive maps, or quantum operations summing to a trace-preserving map). In this paper, I will generalize the projection postulate to POVM's. There are many collections of operations which are consistent with a given measurement. I show that this generalized "projection" postulate selects the set of quantum operations which is, on average, least disturbing to an initially completely unknown input state. I then investigate the tradeoff between information gained in a measurement, and expected disturbance of a completely unknown initial state. This tradeoff is a quantitative expression of one of the most salient and distinctive features of quantum mechanics: that measurement disturbs a quantum mechanical system. 


\section{QUANTUM MEASUREMENTS AND OPERATIONS}

A very general characterization of physically realizable measurement processes is: allow the system to be measured to interact unitarily with another system, often termed the "ancilla", which starts out in some standard state. Then measure some set of orthogonal projectors on the ancilla. The outcomes of this measurement may provide information about the system, and therefore may be considered to be the results of a measurement on the system. There is no need to consider the effect of this measurement on the ancilla if one is only interested in the system, for whether the projection postulate, or some other rule, describes what happens to the ancilla, is not relevant to what happens to the system. The probabilities of the various results of this measurement, and the associated change in the system density operator, may be described solely in terms of the system itself, via the formalism of Positive Operator Valued Measures (POVM's) and associated "operations".

A discrete POVM is set of positive operators $F_{b}$ indexed by positive integers, say, such that

$$
\sum_{b} F_{b}=I
$$

and the probability of obtaining the measurement result with index $b$ is $\operatorname{Tr} \rho F_{b}$. For a standard measurement of a Hermitian observable on the system, the $F_{b}$ are just the projectors onto the eigenspaces of the observable. Such a measurement of projectors is often called "projectionvalued" (not to be confused with a "projective" measurement as defined above). I will often call the elements $F_{b}$ of a POVM "effects," following Ludwig [7] and Kraus [8]. We will also have some occasion to use continuously indexed POVM's, corresponding to a continuum of possible measurement results. These may be loosely thought of as a continuously indexed set of "infinitesimal" positive operators $d \mu(\alpha) F_{\alpha}$, such that $\int d \mu_{\alpha} F_{\alpha}=I$. The probability that $\alpha$ lies in a Borel set $\Delta$ is then given by $\left.\operatorname{tr} \rho \int_{\Delta} d \mu(\alpha) F_{\{} \alpha\right\}$.

I believe that confining our attention to discrete, indeed finitely indexed, POVMs and instruments results in no loss of generality. Arguments similar to, but more involved than, those of Davies [9] and of Ozawa [10] (who treat the maximal information without a disturbance constraint) should show that since the optimal information for a given disturbance can always be achieved with a POVM having a finite number of outcomes (bounded in advance by a polynomial in the dimension of Hilbert space) even when we initially vary over more general sets of physically reasonable POVMs. Since this promises to be rather technical, it will be worked out elsewhere. Nevertheless, in Section VII it will be useful to use a continuously indexed POVM rather than discrete ones achieving the same information-disturbance combination, because of the continuous POVM's greater symmetry.

The general form for the post-measurement quantum state (density operator) conditional on obtaining the result $b$ for a measurement of a POVM consisting of operators $F_{b}$ is [11] [8] |12]:

$$
\rho_{b}^{\prime}=\mathcal{A}_{b}(\rho)=\sum_{i} A_{b i} \rho A_{b i}^{\dagger},
$$

where the $A_{b i}$ satisfy

$$
\sum_{i} A_{b i}^{\dagger} A_{b i}=F_{b}
$$


The linear map $\mathcal{A}_{b}$, often referred to as an operation, will be said to have a Hellwig-Kraus (HK) decomposition, or simply a decomposition, $\left\{\mathcal{A}_{b i}\right\}$; I will often write this $\mathcal{A}_{b} \sim\left\{A_{b i}\right\}_{i}$. Note that here and below I use a convention for ensembles or sets denoted by expressions within curly brackets. The convention is that when we put part of the expression within the brackets as a subscript of the right-hand bracket, the overall expression refers to the ensemble given by the expression within brackets, when only the subscripted piece varies. Thus for example $\left\{\rho_{i j}\right\}$ refers to the ensemble of the $\rho_{i j}$ for various $j$ and fixed $i$. This is, therefore, the $i$-th in a list of ensembles indexed by $i$. (Somewhat irregularly, when there would only be one subscript and it already appears as the sole subscript of the expression within brackets, I will omit it outside the brackets; thus $\left\{F_{b}\right\}$ means $\left\{F_{b}\right\}_{b}$.) I will sometimes refer to the operators of a decomposition as HK operators.

Define $\mathcal{A}:=\sum_{b} \mathcal{A}_{b}$, (so that $\mathcal{A}(\rho)=\sum_{b i} A_{b i} \rho A_{b i}^{\dagger}$. This is the overall operation if one does not know the measurement result; it $\mathcal{A}$ is trace-preserving. Notice that $\rho_{b}^{\prime}$ is unnormalized, and its trace gives the probability of the measurement outcome. As usual, I denote a normalized version of an operator with a hat:

$$
\hat{\rho}_{b}^{\prime}:=\rho_{b}^{\prime} / \operatorname{tr} \rho_{b}^{\prime}
$$

I will say that an operation $\mathcal{A}$ is compatible with a POVM $\left\{F_{b}\right\}$ if there exists an HK decomposition $\left\{A_{b i}\right\}$ of $\mathcal{A}$ such that (3) holds. The collection of operations $\mathcal{A}_{b}$ defined by $\mathcal{A}_{b} \sim\left\{A_{b i}\right\}_{i}$ is often referred to as an instrument for the POVM [13]. When an operation $\mathcal{A}$ is viewed as an instrument for a compatible POVM $\Sigma=\left\{F_{b}\right\}$, I will sometimes call this the procedure $(\Sigma, \mathcal{A})$; this is equivalent to the instrument $\left\{\mathcal{A}_{b}\right\}$. If we use the polar decomposition $A_{b i}=U_{b i} P_{b i},(P$ positive, $U$ unitary $)$, then we have that $F_{b}=\sum_{i} P_{b i}^{2}$. If $P_{b i}$ does not vary with $i$, then all the $P_{b i}$ are proportional to $F_{b}^{1 / 2}$, and with $b$ known the value of $i$ contains no additional information about the initial state. If $P_{b i}$ does vary with $i$, then the value of $i$ represents further information that is not gathered by the POVM $\left\{F_{b}\right\}$, but which could have been gathered via a POVM $\left\{P_{b i}^{2}\right\}$ consistent with the same operation. In fact, one can construct a physical realization of this operation (unitary evolution on system plus ancilla followed by projective measurement on the ancilla) such that measuring $F_{b}$ instead of $P_{b i}^{2}$ just corresponds to coarse-graining the projective measurement on the ancilla by grouping projectors together to form higher-dimensional ones. One might expect that the potential for gathering more information will remove more quantum coherence, and result in more disturbance of the post-system state. The $U_{b i}$ may be thought of as unitary operations that the system undergoes conditional on measurement outcomes $b$ and (if they vary with $i$ ) on potential measurement outcomes $i$ which are not gathered by the POVM $\left\{F_{b}\right\}$ but which are nevertheless available to the apparatus, so that the further evolution of the state may be conditioned on them. If the $U_{b i}$ vary with $i$ while $P_{b i}$ does not, then these further "potential measurement outcomes" carry no information about the pre-measurement system state, and simply represent a stochastic resetting of the state which is not conditioned on any further information about the state - a further noisy disturbance of the state.

A natural generalization of a projective measurement is to have a single value of $i$ in the above sum, and let $A_{b i}=F_{b}^{1 / 2}$, so that the unnormalized conditional density operator and the unconditional post-measurement density operator are given by:

$$
\rho_{b}^{\prime}=F_{b}^{1 / 2} \rho F_{b}^{1 / 2}
$$




$$
\rho^{\prime}=\sum_{b} F_{b}^{1 / 2} \rho F_{b}^{1 / 2}
$$

I will say that such measurement procedures exhibit "the square-root conditional dynamics," and call the associated operation the square-root operation for that measurement. Sometimes I will call this "the square-root measurement procedure," although care should be taken not to confuse this with the "pretty-good measurement", which some authors [14] call the "square-root measurement". In part because of the polar decomposition of the $A$ 's just discussed, we may view any measurement of $\left\{F_{b}\right\}$ as beginning with the performance of the square root conditional dynamics, followed, possibly, by further conditional operations; this provides one (rather weak) motivation for thinking of the square root dynamics as the "minimal disturbance" one is compelled to cause. (It is a weak motivation because the subsequent conditional dynamics can, for some ensembles, be chosen to on average repair some of the square root measurement's damage to the initial state.) Even in the case of projection-valued measures, the square-root operation is a very special case, in which the unitaries $U_{b i}$ are all the identity $I$ (up to an irrelevant phase) and for each $b$ there is only one $A_{b}$, which in this case will just be the projector corresponding to the measurement outcome. None of the freedom to add noise by further conditional unitary operations, or to further disturb the state by effectively collecting extra information which is then thrown away, is used in a square-root measurement procedure.

\section{DISTURBANCE MEASURES AND THE INFORMATION-DISTURBANCE FRONTIER}

In light of the general formulation of quantum measurement and its effect on a system, the question arises: is there anything special about the projection postulate, and more generally about the Lüders type of measurement? It is sometimes said, in the context of nondegenerate Hermitian observables, that it is the "least disturbing" type of measurement, since when the measurement is immediately repeated, one gets the same value of the observable with certainty. However, this only means that it doesn't disturb its own eigenstates. Other states certainly are disturbed, by projection onto the eigenstates of the observable, and it behooves us to ask whether this disturbance is in any sense minimal. If so, one would also like to know whether $F_{b}^{1 / 2}$ is the minimal-disturbance generalization to POVMs. There, it is no longer necessarily true that repeating the measurement is guaranteed to give the same result when the operation is $F_{b}^{1 / 2}$. (There is no conditional dynamics which can provide this guarantee in the case of nonorthogonal $F_{b}$.)

I will use the fidelity $F(\rho, \sigma):=\left(\operatorname{tr} \sqrt{\rho^{1 / 2} \sigma \rho^{1 / 2}}\right)^{2}$ [15], [16], [17, in specifying a measure of disturbance for quantum states. For pure states $\rho=|\psi\rangle\langle\psi|$, this is just $\langle\psi|\sigma| \psi\rangle$. It is unity when $\rho=\sigma$, and zero when their supports are orthogonal. It is therefore a reasonable measure of how similar two quantum states are. We may define $1-F(\rho, \mathcal{A}(\rho))$ to be the disturbance to the state $\rho$ by a measurement procedure resulting in the operation $\mathcal{A}=\sum_{b} \mathcal{A}_{b}$ :

$$
D:=1-F\left(\rho, \sum_{b} \mathcal{A}_{b}(\rho)\right) .
$$

Given an ensemble of density operators $\left\{\rho_{\alpha}, \mu(\alpha)\right\}_{\alpha}$, there are several ways one might construct a measure of the average disturbance caused by measurement. For example, one 
might consider one minus the ensemble average fidelity to the input density operator, of the post-measurement density operator obtained from each ensemble member by averaging over measurement results:

$$
\bar{D}_{1}:=1-\int d \mu(\alpha) F\left(\rho_{\alpha}, \mathcal{A}\left(\rho_{\alpha}\right)\right)
$$

More reasonable in the context of measurement might be consider the fidelity of input density operators to the output density operator $\mathcal{A}_{b}\left(\rho_{\alpha}\right)$ conditional on the measurement result $b$, averaged over both the input ensemble and the measurement result:

$$
\bar{D}_{2}:=1-\int d \mu(\alpha) \sum_{b} F\left(\rho_{\alpha}, \mathcal{A}_{b}\left(\rho_{\alpha}\right)\right)
$$

This is disturbance from the point of view of someone carrying out the measurement, or apprised of its result; the previous quantity is from the point of view of an outside observer who does not know the result. Since $F$ is not linear, these do not define the same quantity; by the concavity of fidelity [17], $\bar{D}_{2} \geq \bar{D}_{1}$. One might also consider the disturbance measures obtained by replacing the first argument of the fidelity function, $\rho_{\alpha}$ in the above formulae, by the ensemble average density operator $\int d \mu(\alpha) \rho_{\alpha}$. These measures seem much less natural (and, again by concavity, each is less than the corresponding one of $\bar{D}_{1}, \bar{D}_{2}$ ). For the case of ensembles of pure input states $\left(\rho_{\alpha}\right.$ pure), $\bar{D}_{1}$ and $\bar{D}_{2}$ coincide. For the rest of this paper, I will consider pure input states, and use this disturbance measure. This is also the measure used by Fuchs and Peres 18 .

The average disturbance to an initial pure state, where the average is taken over some ensemble of pure states specified by a probability measure $\mu(|\psi\rangle)$, on Hilbert space, is given by

$$
\begin{array}{r}
\bar{D}:=1-\int d \mu(|\psi\rangle) \sum_{b} F\left(\mathcal{A}_{b}(|\psi\rangle\langle\psi|),|\psi\rangle\langle\psi|\right) \\
=1-\int d \mu(\psi) \sum_{b i}\left|\left\langle\psi\left|A_{b i}\right| \psi\right\rangle\right|^{2}
\end{array}
$$

The ensemble I will be most concerned with is $d \mu(|\psi\rangle)=d \Omega_{\psi}$, the unitarily invariant measure on Hilbert space, normalized to integrate to 1.

To measure the information gained about an initial ensemble $\Psi \sim d \mu(|\psi\rangle)$, I will use the mutual information between the prior distribution and the measurement outcome, denoted $H(\Psi: B)$. Note that $\Psi$ is a random variable taking Hilbert space vectors as values, and distributed according to $d \mu(|\psi\rangle) ; B$ is a random variable taking measurement results $b$ as values, distributed according to $p(b|| \psi\rangle)=\operatorname{tr} F_{b}|\psi\rangle\langle\psi|=\left\langle\psi\left|F_{b}\right| \psi\right\rangle$, conditional on the initial state $|\psi\rangle$. The information gain is:

$$
H(B: \Psi)=H(B)-H(B \mid \Psi) .
$$

The second term is the average, using the measure $d \mu(|\psi\rangle)$ over states $|\psi\rangle$, of the conditional information

$$
\left.H(B|| \psi\rangle):=-\sum_{b} p(b|| \psi\rangle\right) \log p(b|| \psi\rangle
$$


I will also occasionally consider a different measure of disturbance, involving the entanglement fidelity

$$
F_{e}(\rho, \mathcal{A}):=\sum_{b i}\left|\operatorname{tr} A_{b i} \rho\right|^{2}
$$

The entanglement fidelity of a density operator $\rho$ under an operation $\mathcal{A}$ is less than or equal to the average pure-state fidelity of any ensemble for $\rho$ under $\mathcal{A}$ [19]. I will define the entanglement disturbance $D_{e}(\rho, \mathcal{A})$ to be $1-F_{e}(\rho, \mathcal{A})$. It is an upper bound to the pure-state disturbance to any ensemble for the density operator $\rho$. Since it is defined for an initial density operator rather than an initial ensemble, it is less suitable than (6) for use in a information-disturbance relation like that described above, since the information gain against which disturbance is graphed involves a particular initial ensemble. However, it does provide a lower bound to the information-disturbance frontier. (We could of course just fix some ensemble, such as the "Scrooge" ensemble for the density operator $\rho$ [20], which is the one about which the minimum information is gained, and plot information gain against minimum entanglement disturbance for this particular fidelity measure. One might speculate that the entanglement disturbance would provide a reasonably tight bound on the disturbance to the Scrooge ensemble.)

Given a measurement and a known initial state $\left|\psi_{0}\right\rangle$, it is easy to come up with an operation, consistent with the measurement, which minimizes the pure-state disturbance (6): just set the state back to its initial value no matter what. This may be accomplished by letting $A_{b i}=\lambda_{b i}\left|\psi_{0}\right\rangle\langle b i\rangle$, where $\lambda_{b i}$ and $|b i\rangle$ are the eigenvalues and eigenvectors of $F_{b}^{1 / 2}$. (It is easily checked that this measurement has average fidelity one, and satisfies the criterion (3) for compatibility with the POVM $\left\{F_{b}\right\}$.) But this measurement will severely disturb other initial states. When we set up our measuring apparatus we may or may not know anything about the states we are going to be measuring. A fair way of assessing whether an operation corresponding to a set of effects is minimally disturbing, without assuming any prior knowledge about the state to be measured, is to minimize the disturbance averaged over initial pure states with the unitarily invariant measure. This also makes the problem of finding the least disturbing measurement analytically tractable.

Ultimately, one would like to find the information-disturbance frontier for a given ensemble, defined as the graph of minimal disturbance for a given amount of information collected about the initial state, against information collected. (We could equivalently define it via the dual optimization problem, as the graph of maximal information collectable by a measurement causing no more than a fixed amount of disturbance, against that disturbance.) Formally, we must define this graph as the infimum of disturbance for a given amount of information collected about the state, and show that this infimum is in fact attainable.

Short of an explicit expression (which seems unlikely for a general ensemble), one would like to derive general properties of this frontier - such as the fact that minimal disturbance increases with information collected. This may appear obvious: one could argue that we couldn't cause less disturbance by collecting more information, for then one could just collect the smaller amount of information by doing an experiment that would collect more information with less disturbance, but adding noise to the readout, or not looking at all details of the answer. Fuchs and Peres [18 have explored this frontier for two-state ensembles, with possible applications to quantum cryptography. 
Some progress toward the structure of the information-disturbance frontier may be made by noting that both disturbance measures considered above (and indeed also all the disturbance measures which are one minus an average ensemble fidelity), are linear in the operation, and the information is linear in the POVM. More precisely, from a set of POVM's $\left\{F_{b}^{i}\right\}$ (where $i$ indexes which POVM and $b$ indexes which operator in the POVM) and associated sets of tracepreserving operations $\left\{\mathcal{A}^{i}\right\}$ indexed by $i$ with operator decompositions $\left\{A_{b}^{i}\right\}$ we construct the POVM's and operations which are convex combinations of these:

$$
\begin{aligned}
\left\{G_{i b}\right\} & :=\left\{\lambda^{i} F_{b}^{i}\right\}, \\
\mathcal{B} & \sim\left\{\sqrt{\lambda^{i}} A_{b}^{i}\right\} .
\end{aligned}
$$

Then for any of the disturbance measures discussed above $\left(1-F_{e}(\rho, \mathcal{A})\right.$ and $1-\bar{F}(E, \mathcal{A})$, regardless of the density operator $\rho$ or the ensemble $E$ used in the average), we have

$$
D(\mathcal{B})=\sum_{i} \lambda^{i} D\left(\mathcal{A}^{i}\right) .
$$

Also, for any ensemble of states:

$$
\bar{H}\left(\left\{G_{i b}\right\}\right)=\sum_{i} \lambda^{i} \bar{H}\left(\left\{F_{b}^{i}\right\}\right) .
$$

where the overbar indicates the ensemble average over the information conditional on the input state. Hence, given any two points in the information-disturbance feasible set, the line joining them is entirely within the set. This implies

Theorem 1 The information-disturbance frontier $D(I)$ for a pure-state ensemble is convex.

(Our convention is that a function $f$ is convex if $\lambda f(x)+(1-\lambda) f(y) \geq f(\lambda x+(1-\lambda) y$ ), i.e. the average of the function is greater than or equal to the function of the average.)

Since the disturbance measures under consideration are positive, and one endpoint of $D(I)$ is at the origin, this implies that the information-disturbance frontier for a pure-state ensemble is nondecreasing: minimal disturbance is nondecreasing with information obtained. That is,

Proposition 2 For any disturbance measure of the form $1-\bar{F}(E, \mathcal{A})$ or $1-F_{e}(\rho, \mathcal{A})$ the minimal disturbance required to obtain a given amount of information about some fixed ensemble (which need not even be that used in the disturbance measure) is nondecreasing in the amount of information obtained. In fact, it may have a flat section following the zeroinformation endpoint, but at some point must become and remain monotonically increasing.

We may use this fact to show:

Proposition 3 For any pure-state ensemble, if a point on the upward-sloping portion of the information-disturbance frontier is attainable then it is attainable by a POVM $\left\{F_{b}\right\}$ measured in such a way that the conditional operations $\left\{\mathcal{A}_{b}\right\}$ may be taken to have a oneoperator decomposition. 
We will say such a measurement procedure has one-term conditional dynamics.

Proof: Consider an operation $\mathcal{A}$ compatible with a POVM $\Sigma$, and suppose that the procedure $(\Sigma, \mathcal{A})$ achieves some point $\zeta$ on the upward-sloping portion of the informationdisturbance frontier. Let $(\Sigma, \mathcal{A})$ have multiterm conditional dynamics. Then $\mathcal{A}$ is also compatible with some POVM $\Upsilon$ which finegrains $\Sigma$, such that $(\Upsilon, \mathcal{A})$ exhibits one-term conditional dynamics. $\Upsilon$ gathers no less information than $\Sigma$. If it gathers the same amount, then $\zeta$ is achievable by $(\Upsilon, \mathcal{A})$ and the proposition is established for the point $\zeta$. If it gathers more information than $\Sigma$, then by the strict monotonicity of this portion of the frontier (Proposition 2), $\mathcal{A}$ must have disturbance greater than the minimal disturbance for $\Sigma$, contradicting the assumption that it was least-disturbing for $\Sigma$, and so establishing the proposition.

So in investigating measurement procedures achieving the information-disturbance frontier, we may confine our attention to those with a single $A_{b}$ for each POVM element $F_{b}$.

In fact, we can also show that for any feasible information-disturbance combination $(D, I)$, there exist ways of achieving $\left(D, I^{\prime}\right)$ and $\left(D^{\prime}, I\right)$ with one-term conditional dynamics, where $D^{\prime}<D$ and $I^{\prime}>I$. The first is done by considering the fine-grained POVM of the proof above; the second by mixing this with the trivial POVM, $I$, measured with oneterm conditional dynamics. This enables us to confine our attention, when considering the form of the information-disturbance frontier, to measurement procedures exhibiting oneterm conditional dynamics, even without any assumption that the frontier is attainable.

However, one might also wish to directly show the superiority of the single-term operations for arbitrary POVM's, and possibly even for ensembles other than the uniform one. That is, one might hope to show

Possibility 4 For any POVM and any pure state ensemble, the set of operations leastdisturbing to that ensemble and compatible with that POVM contains an operation with one-term conditional dynamics.

One might even try to show that the least-disturbing operations compatible with a POVM all have one-term conditional dynamics. (To show this, the definition of one-term conditional dynamics would have to modified so as to include, at least, "trivial" multiterm conditional dynamics in which the many Kraus operators $A_{b i}$ have, when polar decomposed, the same isometric part, and positive parts proportional to each other.) Multiple-term operations consistent with the same POVM involve potentially collecting more information, and so it seems reasonable that this would cause more disturbance. Conceivably, however, it might cause less disturbance if the additional information helped restore the initial state better than could be done without it.

It appears difficult to establish the desired property in general, but we may show it for the uniform ensemble. (It is easy to show if our disturbance measure, instead of an ensemble average fidelity, is one minus the entanglement fidelity of the uniform density operator; this is done in Appendix A.)

Theorem 5 One-term conditional dynamics always give a minimally-disturbing way of measuring a given POVM, on the uniform ensemble.

Consider the contribution to $\bar{F}$ from a particular value of $b$ : 


$$
\sum_{i} \int d \Omega_{\psi}\left|\left\langle\psi\left|A_{b i}\right| \psi\right\rangle\right|^{2} \equiv \int d \Omega_{\psi} \mid\left\langle\psi\left|\mathcal{A}_{b}(|\psi\rangle\langle\psi|)\right| \psi\right\rangle .
$$

The disturbance in the multi-term case separates into terms for each $A_{b i} \equiv U_{b i} P_{b i}$ in which $i$ indexes the different operators corresponding to the outcome $b, U_{b i}$ is unitary and $P_{b i}$ positive (the polar decomposition again). From this and the result of [4] (cf. [21]) that for $X \geq 0$, $\max _{\text {unitary } V}|\operatorname{tr} V X|$ occurs where $V X=\sqrt{X^{\dagger} X}$, it follows that $\left.\left|A_{b i}\right| \psi\right\rangle\left.\right|^{2}$ is maximized where $U_{b i}=I$, so $A_{b i}=P_{b i}^{1 / 2}$.

We therefore proceed by a proposition which will be proved below.

Proposition 6 For any $|\psi\rangle$ and positive $P_{1}, P_{2}$

$$
\left\langle\psi\left|P_{1}\right| \psi\right\rangle^{2}+\left\langle\psi\left|P_{2}\right| \psi\right\rangle^{2} \leq\left\langle\psi\left|\sqrt{P_{1}^{2}+P_{2}^{2}}\right| \psi\right\rangle^{2} .
$$

This implies that $A_{b}=\sqrt{F_{b}}$ is a minimally disturbing operation to $\Omega$ for general POVM's, since any (finite) purportedly better set of operations can be repeatedly coarse-grained in the manner of Equation 18 to arrive at $\sqrt{F_{b}} \equiv \sqrt{\sum_{b i} P_{b i}^{2}}$. This proves Theorem 5 .

In fact, Proposition (6), implies that for any initial ensemble, not just the uniform one, coarse-graining the measurement decreases the disturbance caused by a measuring with square-root conditional dynamics. However, this does not yet prove that coarse-graining a measurement decreases the minimal disturbance for an arbitrary ensemble, for the minimally disturbing operation compatible with a given POVM will generally not be the square-root operation unless the ensemble is uniform.

For our application, we also have $\sqrt{P_{1}^{2}+P_{2}^{2}} \leq I$, but the proposition holds more generally. Proposition 6 is not hard to prove when the $P_{b i}$ commute. Let $P_{1}$ have (positive) eigenvalues $\lambda_{i}$. Let $P_{2}$ have (positive) eigenvalues $\eta_{i}$ for the same eigenvectors as $P_{1}$, so that they commute. Then $\sqrt{P_{1}^{2}+P_{2}^{2}}$ commutes with them, and has positive eigenvalues $\sqrt{\lambda_{i}^{2}+\eta_{i}^{2}}$ and the same eigenvectors. We will use these eigenvectors as a basis and write the inequality in components, with $x_{i}$ being the $i$-th component of $|\psi\rangle$ in this basis. The desired inequality (18) becomes:

$$
\begin{array}{r}
\left(\sum_{i} x_{i}^{2} \lambda_{i}\right)^{2}+\left(\sum_{i} x_{i}^{2} \eta_{i}\right)^{2} \leq\left(\sum_{i} x_{i}^{2} \sqrt{\lambda_{i}^{2}+\eta_{i}^{2}}\right)^{2} \\
\sum_{i j} x_{i}^{2} x_{j}^{2} \lambda_{i} \lambda_{j}+\sum_{i j} x_{i}^{2} x_{j}^{2} \eta_{i} \eta_{j} \\
\leq \sum_{i j} x_{i}^{2} x_{j}^{2} \sqrt{\left(\lambda_{i}^{2}+\eta_{i}^{2}\right)\left(\lambda_{j}^{2}+\eta_{j}^{2}\right)} \\
\sum_{i j} x_{i}^{2} x_{j}^{2}\left(\lambda_{i} \lambda_{j}+\eta_{i} \eta_{j}\right) \\
\leq \sum_{i j} x_{i}^{2} x_{j}^{2} \sqrt{\lambda_{i}^{2} \lambda_{j}^{2}+\eta_{i}^{2} \eta_{j}^{2}+\lambda_{i}^{2} \eta_{j}^{2}+\lambda_{j}^{2} \eta_{i}^{2}} .
\end{array}
$$

Rewriting the LHS as

$$
\begin{array}{r}
\sum_{i j} x_{i}^{2} x_{j}^{2} \sqrt{\left(\eta_{i} \eta_{j}+\lambda_{i} \lambda_{j}\right)^{2}} \\
=\sum_{i j} x_{i}^{2} x_{j}^{2} \sqrt{\lambda_{i}^{2} \lambda_{j}^{2}+\eta_{i}^{2} \eta_{j}^{2}+2 \lambda_{i} \lambda_{j} \eta_{i} \eta_{j}} .
\end{array}
$$


we see that if

$$
\lambda_{i}^{2} \eta_{j}^{2}+\lambda_{j}^{2} \eta_{i}^{2} \geq 2 \lambda_{i} \lambda_{j} \eta_{i} \eta_{j}
$$

then the LHS is less than the RHS. And this is indeed the case: letting $a=\lambda_{i} \eta_{j}$ and $b=\lambda_{j} \eta_{i}$, it reduces to the fact that $a^{2}+b^{2} \geq 2 a b$ (which is true since $(a-b)^{2} \geq 0$, with equality iff $a=b)$. Equality in our expression occurs when $\lambda_{i} \eta_{j}=\lambda_{j} \eta_{i}$ for all $i, j$, that is, when $\lambda_{i} / \lambda_{j}=\eta_{i} / \eta_{j}$. In other words, the POVM elements $P_{b i}^{2}$ are proportional to each other. This implies that knowing which of them occured gives us no additional information about the state.

Proof of Proposition [6: In the general case Proposition 6 follows quickly from the following theorem of T. Ando [22], which is easily seen to be equivalent to Lieb's concavity theorem ( [23]; see also discussions in [24], especially p. 273, and [25]).

Theorem 7 (Ando) For $0 \leq t \leq 1$, the map:

$$
(A, B) \rightarrow A^{t} \otimes B^{1-t}
$$

is jointly concave on pairs of positive operators $A, B$.

Proof of Proposition 6: Consider the map from operators to the reals given by:

$$
\mathcal{F}(A)=\left\langle\psi\left|A^{1 / 2}\right| \psi\right\rangle^{2} .
$$

Then (18) is equivalent to the superadditivity of $\mathcal{F}: \mathcal{F}(A)+\mathcal{F}(B)=\mathcal{F}(A+B)$ on the cone of positive operators (let $A=P_{1}^{2}, B=P_{2}^{2}$ ). Since $\mathcal{F}$ is linearly homogeneous $(\mathcal{F}(\lambda A)=\lambda \mathcal{F}(A)$,$) this is equivalent to the concavity of \mathcal{F}$ on the unit interval. Also, $\mathcal{F}(A) \equiv\left\langle\psi\left|\left\langle\psi\left|A^{1 / 2} \otimes A^{1 / 2}\right| \psi\right\rangle\right| \psi\right\rangle$. Ando's theorem has as a special case the concavity of the mapping $A \rightarrow A^{1 / 2} \otimes A^{1 / 2}$, which implies that any diagonal matrix element of it (in any basis) including that between $|\psi\rangle|\psi\rangle$ and itself, is a concave function. (Ando's theorem holds on the entire cone of positive operators, which is why we did not need the restriction $\sqrt{P_{1}^{2}+P_{2}^{2}} \leq I$ in Proposition 6.)

\section{MINIMALLY-DISTURBING OPERATIONS COMPATIBLE WITH A GIVEN MEASUREMENT}

With arbitrary POVMs, with the operation for each measurement outcome given by a single decomposition operator $A_{b}$, we can show that $A_{b}=F_{b}^{1 / 2}$ is a minimal-disturbance operation and evaluate the minimal disturbance. That is,

Theorem 8 Let $\left\{F_{b}\right\}$ be a POVM, and let $\left\{\mathcal{A}_{b}\right\}$ be a set of operations compatible with that POVM. If each $\mathcal{A}_{b}$ has an operator decomposition consisting of a single operator, then

$$
\begin{aligned}
\sum_{b} & \int d \Omega_{\psi} F\left(|\psi\rangle\langle\psi|, \mathcal{A}_{b}(|\psi\rangle\langle\psi|)\right) \\
& \leq \sum_{b} \int d \Omega_{\psi} F\left(|\psi\rangle\left\langle\psi\left|, F_{b}^{1 / 2}\right| \psi\right\rangle\langle\psi| F_{b}^{1 / 2}\right) \\
& =\frac{1}{d(d+1)}\left(d+\sum_{b}\left(\operatorname{tr} F_{b}^{1 / 2}\right)^{2} .\right.
\end{aligned}
$$


The proof proceeds via the following Lemma, which also appears with a different proof in [26].

Lemma 9 Define

$$
\Pi:=\int d \Omega_{\psi}|\psi\rangle\langle\psi|\otimes| \psi\rangle\langle\psi|
$$

Then

$$
\Pi=\frac{1}{d(d+1)} \sum_{i j}|i\rangle\langle i|\otimes| j\rangle\langle j|+| i\rangle\langle j|\otimes| j\rangle\langle i|
$$

Proof of Lemma:

$$
\begin{array}{r}
\int d \Omega_{\psi}|\psi\rangle\langle\psi|\otimes| \psi\rangle\langle\psi| \\
=\int d \Omega_{\psi} \sum_{i j l m}\langle i \mid \psi\rangle\langle\psi \mid j\rangle\langle l \mid \psi\rangle\langle\psi \mid m\rangle|i\rangle\langle j|\otimes| l\rangle\langle m| .
\end{array}
$$

With the notation $\langle i \mid \psi\rangle=r_{i} e^{i \phi_{i}}$, etc..., the $i j l m$-th matrix element of $\Pi$ may be written as:

$$
\int d \boldsymbol{r} d \boldsymbol{\phi} \delta(|\boldsymbol{r}|-1) r_{i} e^{i \phi_{i}} r_{j} e^{-i \phi_{j}} r_{l} e^{i \phi_{l}} r_{m} e^{-i \phi_{m}} .
$$

Here $d \boldsymbol{r}=d r_{1} d r_{2} \cdots d r_{d}, d \boldsymbol{\phi}=d \phi_{1} \cdots d \phi_{d}$.

The angular integrals give zero except in three cases, for which the matrix elements in (28) are as follows:

$$
\begin{array}{ll}
\text { 1. } i=j, l=m, i \neq l: & \int d \Omega_{\psi}|\langle i \mid \psi\rangle|^{2}|\langle l \mid \psi\rangle|^{2} \\
\text { 2. } i=m, j=l, i \neq j: & \int d \Omega_{\psi}|\langle i \mid \psi\rangle|^{2}|\langle j \mid \psi\rangle|^{2} \\
\text { 3. } i=j=l=m: & \int d \Omega_{\psi}|\langle i \mid \psi\rangle|^{4} .
\end{array}
$$

The integrals are easily done using Eq. (12) of Jones [27], which yields:

$$
\int d \Omega_{\psi}|\langle\psi \mid a\rangle|^{2}|\langle\psi \mid b\rangle|^{2}=\frac{1+|\langle a \mid b\rangle|^{2}}{d(d+1)},
$$

where $|a\rangle,|b\rangle$ are any normalized, but not necessarily orthogonal or identical, vectors. For our cases 1 and 2 , the matrix elements are $1 / d(d+1)$; case 3 gives $2 / d(d+1)$. We combine $1 / 2$ times the case 3 terms with each of case 1 and 2, enabling us to remove the inequality condition on the indices, and change the dummy index $l$ to $j$ to obtain the Lemma. Proof of Theorem \&:

Note that

$$
\int d \Omega_{\psi}\langle\psi|A| \psi\rangle\langle\psi|B| \psi\rangle \equiv \operatorname{tr}(\Pi(A \otimes B)),
$$

Lemma 9 enables one to write this as $(1 / d(d+1)) \sum_{i j}(\langle i|A| i\rangle\langle j|B| j\rangle+\langle i|A| j\rangle\langle j|B| i\rangle)$. Hence the average overlap becomes: 


$$
\begin{aligned}
\frac{1}{d(d+1)} \sum_{b} \sum_{i j} & \left(\left\langle i\left|A_{b}\right| i\right\rangle\left\langle j\left|A_{b}^{\dagger}\right| j\right\rangle+\left\langle i\left|A_{b}\right| j\right\rangle\left\langle j\left|A_{b}^{\dagger}\right| i\right\rangle\right) \\
= & \left.\frac{1}{d(d+1)} \sum_{b}\left(\left|\operatorname{tr} A_{b}\right|^{2}+\operatorname{tr} A_{b} A_{b}^{\dagger}\right)\right) .
\end{aligned}
$$

By the linearity and cyclicity of the trace and the fact that $\mathcal{A}$ is trace-preserving $\left(\sum_{b} A_{b}^{\dagger} A_{b}=\right.$ $I$ ), the second term in parentheses is $d$. We wish to maximize this overlap (thereby minimizing disturbance) over all single-term operations compatible with $F_{b}$. So for each $b$, we maximize the $b$-th term over all $A_{b}$ such that $A_{b}^{\dagger} A_{b}=F_{b}$. By the polar decomposition of operators, such $A_{b}$ have the form $U_{b} F_{b}^{1 / 2}$. From this and the result of [4] (cf. [21]) that for $A \geq 0, \max _{\text {unitary } V}|\operatorname{tr} V A|$ occurs where $V A=\sqrt{A^{\dagger} A}$, it follows that $\left|\operatorname{tr} A_{b}\right|$ is maximized

where $U=I$, so $A_{b}=F_{b}^{1 / 2}$. Thus the optimum overlap is obtained with the square root conditional dynamics. It is given by:

$$
\bar{F}_{\max }=\frac{1}{d(d+1)}\left(d+\sum_{b}\left(\operatorname{tr} F_{b}^{1 / 2}\right)^{2}\right) .
$$

The corresponding minimal disturbance is

$$
\bar{D}_{\min }=1-\bar{F}_{\max } .
$$

Consider the special case of effects proportional to one dimensional projectors. The effects $F_{b}$ become $g_{b}|b\rangle\langle b|$, where $g_{b}$ are proportionality constants satisfying $\sum_{b} g_{b}=d$. The optimum overlap and disturbance for the uniform ensemble, with one-term conditional dynamics, are given by:

$$
\bar{F}_{\text {max }}=\frac{2}{d+1}, \bar{D}_{\text {min }}=\frac{d-1}{d+1} .
$$

\section{INFORMATION}

We have found, in Eq. (35), the minimum disturbance for measurement of an arbitrary POVM. This is a step towards deriving the information-disturbance frontier. As a special case, we found the minimal disturbance to be $(d-1) /(d+1)$ for a class of measurements in which the effects are proportional to one-dimensional projectors. At the opposite pole from these "fine-grained measurements" is the ultimate coarse-grained measurement of a single effect which is the identity operator. This yields zero information, and can be accomplished with no disturbance. These extreme cases presumably represent the endpoints of the information-disturbance frontier. Another step toward deriving the frontier is to find the information gained in measurements of the fine-grained type investigated above, which is clearly greater than zero, as is the disturbance they cause. This will pin down the maximal-information endpoint. It turns out that the information yield is the same for all such fine-grained measurements, whether the effects are orthogonal or not. This is a special case of the fact that any fine-grained measurement gives the same information about the 
"Scrooge" ensemble. (The Scrooge ensemble for a given density operator $\rho$ is defined as the ensemble (from among ensembles for $\rho$ ) for which the accessible information is minimal [20]. The uniform ensemble is the Scrooge ensemble for the uniform density operator $I / d$.)

Here I present a different derivation of the information gained by a finegrained measurement, which applies to the the uniform ensemble only and uses the methods of Jones [27. Recall that the information gain from measurement is the mutual information between the prior distribution and the measurement outcome, denoted $H(\Psi: B)$. I will use this in the form:

$$
H(B: \Psi)=H(B)-H(B \mid \Psi) .
$$

This can be calculaated form the prior probability measure on states $p(|\psi\rangle)$ which we assume to be the unitarily invariant one, and the conditional probabilities $p(b \mid \psi)$ of the data (measurement outcomes) given the initial state, which are $\operatorname{Tr} g_{b}|b\rangle\langle b \mid \psi\rangle\left\langle\left.\psi\left|=g_{b}\right|\langle b \mid \psi\rangle\right|^{2}\right.$. (Here I use the notation for finegrained measurements introduced at the end of Section IV.) The first term is

$$
H(B)=-\sum_{b} p(b) \log p(b)
$$

Since

$$
\begin{gathered}
p(b) \equiv \int d \Omega_{\psi} p(|\psi\rangle) p(b \mid \psi)=\int d \Omega_{\psi} g_{b}|\langle b \mid \psi\rangle|^{2}=\frac{g_{b}}{d}, \\
H(B)=-\sum_{b} \frac{g_{b}}{d} \log \frac{g_{b}}{d}=-\frac{1}{d} \sum_{b} g_{b} \log g_{b}+\log d,
\end{gathered}
$$

where I have used equation (7) of [27] to do the integral, and have also made use of the fact that $\sum_{b} g_{b}=d$.

The second term is:

$$
\begin{aligned}
H(B \mid \Psi) & =-\int d \Omega_{\psi} \sum_{b} p(b \mid \psi) \log p(b \mid \psi) \\
& =-\int d \Omega_{\psi} \sum_{b} g_{b}|\langle b \mid \psi\rangle|^{2} \log g_{b}|\langle b \mid \psi\rangle|^{2} \\
& =-\sum_{b} g_{b} \int d \Omega_{\psi}|\langle b \mid \psi\rangle|^{2}\left(\log g_{b}+\log |\langle b \mid \psi\rangle|^{2}\right) \\
& =-\sum_{b} g_{b} \log g_{b} \int d \Omega_{\psi}|\langle b \mid \psi\rangle|^{2} \\
& -\sum_{b} g_{b} \int d \Omega_{\psi}|\langle b \mid \psi\rangle|^{2} \log |\langle b \mid \psi\rangle|^{2} .
\end{aligned}
$$

The first integral is the same one we encountered in $H(B)$, and its value is $1 / d$. The second integral is more complicated, but can be done using the same formula as the first (or see [28]); its value is 


$$
-\frac{1}{d} \sum_{k=1}^{d-1} \frac{1}{1+k}
$$

Hence

$$
H(B \mid \Psi)=\frac{1}{d} \sum_{b} g_{b} \log g_{b}+\sum_{k=1}^{d-1} \frac{1}{1+k} .
$$

Combining equations (46) and (40), we obtain

$$
H(B: \Psi)=\log d-\sum_{k=1}^{d-1} \frac{1}{1+k} .
$$

This depends only on $d$, and not on the weights $g_{b}$; as long as the $F_{b}$ are proportional to one-dimensional projectors, the information gained about a maximally uncertain initial pure state is the same, whether the measurement is of orthogonal projectors or some other set of maximally fine-grained effects.

Unfortunately, finding the information gain from measuring an arbitrary POVM is a much more difficult problem.

\section{THE INFORMATION-DISTURBANCE FRONTIER}

For the information-disturbance frontier, we need the information gain maximized over possible measurements and compatible operations causing a given level of disturbance (or less). Equivalently, we need the minimal disturbance measurement and associated operation which gives a fixed level of information gain. Since the minimal disturbance associated with all fine-grained measurements is the same, and they all yield the same information gain, we have found the high-information endpoint of the information-disturbance frontier. For any other set of effects will be a blurring (by allowing positive operators not proportional to projectors) or coarsening (by allowing higher-dimensional projectors) of these effects, resulting in less information gain and the possibility of less disturbance. Clearly, the other endpoint is at zero information and zero disturbance, achieved by the identity operation of doing nothing. One might speculate that the minimally disturbing measurement (for the uniform ensemble) for any given level of information obtained, is to measure a fine-grained set of effects with some probability, and otherwise to do nothing. That is, our POVM is given by the set $\left\{\alpha I,(1-\alpha) F_{b}\right\}$, where the $F_{b}$ form a fine-grained POVM. Then the tradeoff frontier is a straight line between the known endpoints. However, it seems unlikely that the frontier is perfectly straight. This would just be too boring to be true. In the next section, we will make some progress towards obtaining a closed form for the information-disturbance frontier, by showing that for each point on the frontier, there exists an optimal measurement procedure associated with a very simple operation, that of swapping in the maximally mixed state with some probability and otherwise leaving the state undisturbed. (This operation is not compatible with the measurement just discussed, that gives the straight-line frontier.) 


\section{TOWARDS THE FULL FRONTIER}

We will say an operation $\mathcal{A}$ is unitarily covariant if

$$
W^{\dagger} \mathcal{A}\left(W \rho W^{\dagger}\right) W=\mathcal{A}(\rho)
$$

for any unitary $W$. We will also introduce a convention for ensembles or sets denoted by expressions within curly brackets. The convention is that when we put part of the expression within the brackets as a subscript of the right-hand bracket, the overall expression refers to the ensemble given by the expression within brackets, when only the subscripted piece varies. Thus for example $\left\{\rho_{i j}\right\}$ refers to the ensemble of the $\rho_{i j}$ for various $j$ and fixed $i$. (This is, therefore, the $i$-th in a list of ensembles indexed by $i$.)

Using the unitary invariance of the ensemble $\Omega$, we will show that

Theorem 10 There is always a unitarily covariant way of obtaining a given I with minimal disturbance to $\Omega$.

In other words, for this measurement and conditional dynamics the operation $\mathcal{A}:=\sum_{b} \mathcal{A}_{b}$ is unitarily covariant.

Proof: By the unitary invariance of the ensemble $\Omega$, for any fixed unitary $U$, the POVM $\left\{U F_{b} U^{\dagger}\right\}$ has the same information and the same minimal disturbance (for $\Omega$ ) as $\left\{F_{b}\right\}$. (This is so because the information depends only on the probabilities $p_{b}=\left\langle\psi\left|U F_{b} U^{\dagger}\right| \psi\right\rangle$, so transforming the POVM is equivalent to transforming the ensemble, which we know is invariant. Similarly, the minimally disturbing conditional dynamics compatible with this POVM are given by the operation with decomposition $A_{b}=\left(U F_{b} U^{\dagger}\right)^{1 / 2} \equiv U F_{b}^{1 / 2} U^{\dagger}$. The average disturbance depends on the $A_{b}$ only through $\left\langle\psi\left|A_{b}\right| \psi\right\rangle \equiv\left\langle\psi\left|U F_{b}^{1 / 2} U^{\dagger}\right| \psi\right\rangle$, so again we may view the unitary transformation as applied to the ensemble, which is invariant under it.) By the linearity of disturbance and information in the POVM and operation, respectively, the continuously indexed POVM

$$
\left\{d \mu(U) U F_{b} U^{\dagger}\right\}_{b, U}
$$

where POVM elements are indexed by both $b$ and $U$, achieves the same information and disturbance as $\left\{E_{b}\right\}$. Here $d \mu(U)$ is the (unitarily invariant) Haar measure on the unitary group $U(d)$. This POVM is unitarily invariant in the sense that applying any unitary $V$ to all elements of the POVM just results in the same POVM with the elements reindexed. The optimal associated operation is given by the continuous decomposition:

$$
\left\{d \mu(U)^{1 / 2} U F_{b}^{1 / 2} U^{\dagger}\right\}_{b, U} .
$$

The "square root of a measure" here is just formal notation. (For a rigorous treatment of such operations as "Radon-Nikodym derivatives of quantum instruments", see [29], [30].) This operation is defined by its action:

$$
\mathcal{A}(\rho)=\sum_{b} \int d \mu(U) U F_{b}^{1 / 2} U^{\dagger} \rho U F_{b}^{1 / 2} U^{\dagger}
$$

note that the formal square root does not appear here. The unitary covariance of $\mathcal{A}$ is straightforward from (51) and the unitary invariance of $d \mu$. 
A unitarily covariant (which we will also call isotropic) operation may be viewed as mixing in the uniform density operator with some probability $p$ (cf. e.g. [31], [32], [33]):

$$
\mathcal{A}_{p}(\rho)=(1-p) \rho+p(I / d) .
$$

This operation causes disturbance

$$
D_{\text {min }}\left(\Omega, \mathcal{A}_{p}\right)=p \frac{d-1}{d} .
$$

To calculate the information-disturbance frontier, we now need only to calculate the maximum, over POVMs compatible with the isotropic operation $\mathcal{A}_{p}$, of the information gathered by the POVM. Here I will not give a closed form for the maximum, but I will give an approach which reduces the problem from a constrained maximization to an unconstrained one. To do this, we recall some more of the basic theory of quantum operations. By saying a POVM is "compatible with the operation" $\mathcal{A}$, we mean that it can be measured by an instrument which gives rise to that operation (when measurement results are averaged over). Any POVM compatible with operation $\mathcal{A}$ is given by coarsegraining some set of operators $\left\{F_{b}\right\}$ defined by $F_{b}=A_{b}^{\dagger} A_{b}$ for some decomposition $\left\{A_{b}\right\}$ of the operation $\mathcal{A}$. We need only consider the POVM's obtained as $\left\{F_{b}:=A_{b}^{\dagger} A_{b}\right\}$, and not the coarsegrainings, since the coarsegrainings obtain less (or at least no more) information. Thus every decomposition of an operation determines a compatible POVM, and all compatible POVM's are obtained by this procedure (plus coarsegraining).

Any two decompositions of the same operation, $\left\{A_{i}\right\}$ having $r$ operators and $\left\{B_{i}\right\}$ having $s$ operators, are related by [12]:

$$
A_{i}=\sum_{j=1}^{s} m_{i j} B_{j}
$$

where $m$ is the matrix of a maximal partial isometry from the complex vector space $\mathcal{C}^{s}$ to $\mathcal{C}^{r}$. A partial isometry is a generalization of a unitary operator, which must satisfy $V V^{\dagger}=\Pi$ for some projector $\Pi$. Such an isometry will then also satisfy $V^{\dagger} V=\Gamma$ for some projector $\Gamma$ having the same rank as $\Pi$. If the range and domain spaces of a linear operator $V$ have different dimensions, it will not be possible to find a unitary mapping between the two: the best one can do is find a partial isometry $V$ such that one of $V V^{\dagger}$ and $V^{\dagger} V$ is the identity (whichever one operates on the smaller space). We will call such a map a maximal partial isometry between the spaces $S_{1}$ and $S_{2}$. A partial isometry with $V V^{\dagger}$ (and hence $V$ ) having rank $C$ may be thought of as projecting onto a $C$-dimensional subspace of $V$ 's domain Hilbert space and then mapping that subspace unitarily to a $C$-dimensional subspace of the range Hilbert space. Thus if $s \leq r$ in (54), $m$ 's columns are $s$ orthonormal vectors in $\mathcal{C}^{r}$ :

$$
\sum_{j} m_{i j}^{*} m_{k j}=\delta_{i k}
$$

or in other words:

$$
m m^{\dagger}=I^{(s)} .
$$

Any quantum operation on a system $Q$ may be realized [34], [11], [8] by a "unitary representation" in which the Hilbert space $Q$ is extended by adjoining an environment $E$ 
prepared in a standard state $\left|0^{E}\right\rangle$, and the system and environment undergo a unitary interaction, followed by a projection on the environment system. Any such unitary interaction with a given initial environment state determines a quantum operation. (In the case of a trace-preserving operation, the environment projection is the identity.) That is,

$$
\mathcal{A}(\rho)=\operatorname{tr}_{E}\left(\pi^{E} U^{Q E}\left|0^{E}\right\rangle\left\langle 0^{E}\right| \otimes \rho^{Q} U^{\dagger Q E} \pi^{E}\right) .
$$

The operators $A_{i}$ in the operator decomposition representation discussed above, turn out to be the "operator matrix elements"

$$
A_{i}^{Q}=\left\langle i^{E}\left|U^{Q E}\right| 0^{E}\right\rangle
$$

of the unitary interaction, between the initial environment state and orthonormal environment vectors $|i\rangle$ of the basis used for the partial trace over the environment. The freedom (54) to "unitarily mix" the operators $A_{i}$, obtaining another valid decomposition, is just the freedom to do the enviroment partial trace in a different environment basis (related to the first by the transpose of the unitary used in remixing). See [19] for a more extended discussion of this. Here, we merely emphasize that in order to get all decompositions as we vary the measurement on the environment, it was assumed that the environment was initially in a pure state.

The import of this for our problem of extracting information about $|\psi\rangle$ via measurements compatible with $\mathcal{A}_{p}$ is that we may vary over the relevant "finegrained" POVMs compatible with $\mathcal{A}_{p}$ by imagining we implement $\mathcal{A}_{p}$ with an initially pure environment, and varying over all measurements on the environment. We may do this by letting the interaction $U^{Q E}$ swap half of bipartite a maximally entangled state from the environment into the system $Q$, conditional on "quantum dice" loaded with probability $p$. 由 Since half (i.e., one subsystem) of a bipartite maximally entangled state has the uniform density operator $I / d$, this just replaces the state of $Q$ with the uniform density operator, with probability $p$. In other words, it effects the isotropic operation with parameter $p$. In more detail, we let the environment be the $\left(d^{2}+1\right)$-dimensional Hilbert space

$$
E=E_{1} \otimes E_{2} \oplus F
$$

where $E_{1} \cong E_{2} \cong Q$ are $d$-dimensional and $F$ is a one-dimensional "flag" on which the swapping is conditioned. We prepare an initial environment state

\footnotetext{
1 "Quantum dice" are usually taken to consist of a pure entangled state of two systems, used as dice by conditioning operations on some third system on the eigenbasis of one of the two entangled systems. The resulting operation on the third system has the effect of randomly performing one of the operations which were performed conditionally, with probabilities given by the eigenvalues of the reduced density matrix of the entangled state. Below we use a slightly different formulation which applies to our special case of either doing or not doing some operation. This involves an extra "flag" dimension of the environment instead of an extra environment qubit. It reduces the required number of Hilbert space dimensions, because we don't have to have the maximally entangled state ready for partial swapping even in that subspace where the swapping won't be done, as we would if we conditioned on a qubit value.
} 


$$
\left|0^{E}\right\rangle=\sqrt{1-p}|F\rangle+\sqrt{p} \sum_{i=1}^{d} \frac{1}{\sqrt{d}}\left|i^{E_{1}}\right\rangle\left|i^{E_{2}}\right\rangle
$$

and realize the operation $\mathcal{A}_{p}$ on $Q$ through the unitary interaction:

$$
U^{Q E}:=\left(S W A P\left(E_{1}, Q\right) \otimes I^{E_{2}}\right) \oplus\left(I^{F} \otimes I^{Q}\right) .
$$

SWAP simply swaps the states of $E_{1}$ and $Q$; it is defined by:

$$
S W A P\left(E_{1}, Q\right)\left|j^{Q}\right\rangle\left|i^{E_{1}}\right\rangle=\left|i^{Q}\right\rangle\left|j^{E_{1}}\right\rangle
$$

so that, overall

$$
\begin{array}{r}
U^{Q E}\left|j^{Q}\right\rangle\left|i^{E_{1}}\right\rangle\left|k^{E_{2}}\right\rangle=\left|i^{Q}\right\rangle\left|j^{E_{1}}\right\rangle\left|k^{E_{2}}\right\rangle \\
U^{Q E}\left|j^{Q}\right\rangle\left|F^{E}\right\rangle=\left|j^{Q}\right\rangle\left|F^{E}\right\rangle .
\end{array}
$$

When $\left|\psi^{Q}\right\rangle$ goes in on the measured system, the final environment state is

$$
\begin{aligned}
& \rho^{E^{\prime}}(|\psi\rangle, p)=(1-p)|F\rangle\left\langle F|+p| \psi^{E_{1}}\right\rangle\left\langle\psi^{E_{1}}\right| \otimes \frac{I^{E_{2}}}{d} \\
& +\sqrt{\frac{(1-p) p}{d}}\left(|F\rangle\left\langle\psi^{E_{1}}\left|\left\langle\psi^{E_{2}}|+| \psi^{E_{1}}\right\rangle\right| \psi^{E_{2}}\right\rangle\langle F|\right)
\end{aligned}
$$

Now, any information about the initial state of $Q$ obtainable by a measurement compatible with $\mathcal{A}_{p}$ may be obtained by measuring the environment $E$ after the above-defined interaction $U^{Q E}$, for each such measurement made on the environment after the interaction corresponds, via the unitary representation of operations, to a decompositions $\left\{A_{b}\right\}$ of the operation $\mathcal{A}_{p}$, and thus to a POVM on $\mathrm{Q}$ compatible with $\mathcal{A}_{p}$, and as we vary over all measurements on an initially pure $E$ we obtain fine-grainings of all such POVM's.

The uniform distribution $\Omega$ for initial states $\psi$ gives rise, via the dynamical evolution $U^{Q E}$, to a distribution $\mu_{p}$ on final environment states $\rho^{E^{\prime}}$. The accessible information about $\rho^{E^{\prime}}$ is the maximal information obtainable about $|\psi\rangle$ by measurements on $E^{\prime}$ consistent with this operation, and hence gives us the maximal information about the initial preparation $|\psi\rangle$ consistent with the isotropic operation $\mathcal{A}_{p}$. As we vary $p$ parametrically, we get the information-disturbance frontier for the uniform pure-state ensemble $\Omega$.

\section{SPHERICAL 2-DESIGNS}

All the results of this paper (notably, Theorems 5 and 8) which involve only average pure-state fidelities over the uniform ensemble (and not, for instance, information), hold also for a class of discrete pure-state ensembles. These ensembles are the spherical t-designs for $t \geq 2$ in $d$-1-dimensional complex projective space $C P_{d-1}$ (that is, the space of rays of the $d$-dimensional Hilbert space, isomorphic to the space of pure quantum states $|\psi\rangle\langle\psi|$ ). Various equivalent definitions of these designs exist, but the one relevant here is that a spherical $t$-design is a finite set $\Delta \subset C P_{d-1}$ such that the uniform integral over $C P_{d-1}$ of a 
polynomial $P$ of degree no higher than $t$ is equal to the discrete average of the polynomial evaluated on the points of the design:

$$
\int_{C P_{d-1}} P(\pi)=\frac{1}{|\Delta|} \sum_{\pi \in \Delta} P(\pi) .
$$

(As usual, $|S|$ denotes the cardinality of a set $S$.) A reasonably good supply of small (size quadratic in the dimension) spherical 2-designs exists, and some are given by the following construction. Define two orthonormal bases to be unbiased [35] or conjugate [36] if any inner product of a vector from one basis with one from the other has modulus $1 / \sqrt{d}$. There exist sets of "complementary" bases which are higher-dimensional analogues of the eigenbases of $\sigma_{x}, \sigma_{y}$, and $\sigma_{z}$. These are the "mutually unbiased bases" (MUBs) introduced by Ivanović [37 (for prime dimension), and by Wootters and Fields [35] (for prime power dimension). Let the index $k=0, \ldots N-1$ specify which basis; $i=1, \ldots, d$ specifies which vector in the basis. A set of $N$ orthonormal bases indexed by $k$ is said to be mutually unbiased [35] or conjugate [36] if for all $k \neq l$

$$
\left|\left\langle e_{i}^{k} \mid e_{j}^{l}\right\rangle\right|=1 / \sqrt{d}
$$

For $d=p^{n}, p$ prime, Wootters and Fields constructed $d+1$ mutually unbiased bases $\left|e_{i}^{k}\right\rangle$. The construction uses the finite field $F_{p^{n}}$ of prime power order, also known as Galois fields $G F\left(p^{n}\right)$, which has $p^{n}$ elements (including zero). For odd primes, the construction is as follows. One basis may be chosen arbitrarily; in this "standard" basis the $l$-th component of the $j$-th vector of the $k$-th basis is:

$$
\left\langle l \mid e_{j}^{k}\right\rangle=\frac{1}{\sqrt{d}} \omega^{\operatorname{Tr}\left[k l^{2}+j l\right]}
$$

where $l, k, j$ range over the $p^{n}$ elements of $F_{p^{n}}$.

$$
\omega:=e^{2 \pi i / p},
$$

(a primitive $p$-th root of unity) and

$$
\operatorname{Tr}[x]:=x+x^{p}+x^{p^{2}}+\cdots+x^{p^{n-1}} .
$$

Note that the trace has values in a subfield of $F_{p^{n}}$ isomorphic to $F_{p}$. Verifying that these are mutually unbiased is a relatively calculation using elementary properties of the trace on finite fields [38], [39]) and Gauss sums [39]. In particular, the properties $\operatorname{Tr}(x+y)=$ $\operatorname{Tr}(x)+\operatorname{Tr}(y),\left(x, y \in F_{p^{n}}\right)$ and $\operatorname{Tr}(c x)=c \operatorname{Tr}(x), c \in F_{p}, x \in F_{p^{n}}$ are fundamental. Wootters and Fields also give a construction for $p=2$, but it is more complicated and I will not present it here. Working independently of Wootters and Fields and of Ivanović, and using ideas from coding theory and finite geometry, Calderbank, Cameron, Kantor, and Seidel [40] also found sets of $d(d+1)$ mutually unbiased bases for prime-power dimension, which may well be the same as Wootters' and Fields'. (At least some cases were also found by other authors cited in [40].) Calderbank et. al. also state that many unitarily inequivalent such sets of MUBs must exist. (Constructions are known at least for $d$ a power of 2.) $d+1$ meets an upper bound (valid for arbitrary $d$ ) on the number of such bases, established by Delsarte, Goethals, and Seidel [41]. I know of no examples meeting the bound for $d$ with distinct prime factors. 
Theorem 11 The set of $d(d+1)$ vectors $\left|e_{i}^{k}\right\rangle$ belonging to the union of the $(d+1)$ mutually unbiased (aka conjugate) bases constructed by Wootters and Fields for $d$ a power of an odd prime, is a spherical 2-design in $C P_{d-1}$.

Proof:

Any second-degree polynomial in $\pi=|\psi\rangle\langle\psi|$ may be written $\sum_{\alpha} \operatorname{tr} \pi A_{\alpha} \operatorname{tr} \pi B_{\alpha}$ for some finite set of linear operators $A_{\alpha}$ and $B_{\alpha}$. (This is shown e.g. in [42], or using Lemma 1 from [43].) So by (32) we need only show that:

$$
\Upsilon:=\frac{1}{d(d+1)} \sum_{k i}\left|e_{i}^{k}\right\rangle\left\langle e_{i}^{k}|\otimes| e_{i}^{k}\right\rangle\left\langle e_{i}^{k}\right|=\Pi .
$$

First consider the operator made by summing over all basis vectors of all the MUBs except the standard basis: $\Lambda:=\sum_{k i \in F_{p^{n}}}\left|e_{i}^{k}\right\rangle\left\langle e_{i}^{k}|\otimes| e_{i}^{k}\right\rangle\left\langle e_{i}^{k}\right|$. In the standard basis this has matrix elements

$$
\begin{aligned}
& \langle\alpha|\langle\gamma|\Lambda| \beta\rangle| \delta\rangle \\
= & \sum_{k i}\left\langle\alpha \mid e_{i}^{k}\right\rangle\left\langle e_{i}^{k} \mid \beta\right\rangle\left\langle\gamma \mid e_{i}^{k}\right\rangle\left\langle e_{i}^{k} \mid \delta\right\rangle \\
= & \left(1 / d^{2}\right) \sum_{k i} \omega^{\operatorname{Tr}\left(k \alpha^{2}+i \alpha\right)-\operatorname{Tr}\left(k \beta^{2}+i \beta\right)+\operatorname{Tr}\left(k \gamma^{2}+i \gamma\right)-\operatorname{Tr}\left(k \alpha^{2}+i \delta\right)} \\
= & \left(1 / d^{2}\right) \sum_{k} \omega^{\operatorname{Tr} k\left(\alpha^{2}-\beta^{2}+\gamma^{2}-\delta^{2}\right)} \sum_{i} \omega^{\operatorname{Tr} i(\alpha-\beta+\gamma-\delta)}
\end{aligned}
$$

We thus have a product of two sums of the form $\sum_{k \in F_{p^{n}}} \omega^{k x}$. This sum is easily shown to be equal to $p^{n} \delta_{x, 0}$. (By definition $\delta_{x, 0}=0$ if $x=0,1$ otherwise.) To show it, note [38 that as $\beta$ ranges over $F_{p^{n}}, \operatorname{Tr} \beta$ takes each value in $F_{p}$ equally often (i.e., $p^{n-1}$ times). As we vary over $k, k x$ for $x \neq 0$ varies over $F_{p^{n}}$ since $f(k): k \mapsto k x$ is a bijection. So we can group the sum into a sum of $p^{n-1}$ copies of $\sum_{\eta \in F_{p}} \omega^{\eta x}=p \delta_{x, 0}$, obtaining overall $p^{n} \delta_{x, 0}$. Thus (72) becomes $\delta_{\alpha^{2}-\beta^{2}+\gamma^{2}-\delta^{2}, 0} \delta_{\alpha-\beta+\gamma-\delta, 0}$. So we have the simultaneous equations:

$$
\alpha^{2}-\beta^{2}+\gamma^{2}-\delta^{2}=0, \alpha-\beta+\gamma-\delta=0
$$

in $F_{p^{n}}$. Rewriting these as

$$
\begin{array}{r}
(\alpha+\beta)(\alpha-\beta)=(\gamma+\delta)(\gamma-\delta) \\
(\alpha-\beta)=(\gamma-\delta)
\end{array}
$$

we see that any $\alpha, \beta, \gamma, \delta$ satisfying $\alpha=\beta, \gamma=\delta$ are solutions, and if one of the latter conditions holds they both do. If $\alpha \neq \beta$ (and so also $\gamma \neq \delta$ ), we can (since our arithmetic is in a field) divide the first equation by the second to get the two equations $\alpha+\beta=\gamma+\delta$, $\alpha-\beta=\gamma-\delta$, which are simultaneously satisfied whenever $\alpha=\gamma, \beta=\delta$. So if we write

$$
\Lambda=\sum \Lambda_{\alpha \gamma \beta \delta}|\alpha\rangle\langle\beta|\otimes| \gamma\rangle\langle\delta|
$$

the matrix elements are

$$
\delta_{\alpha \gamma} \delta_{\beta \delta}+\delta_{\alpha \beta} \delta_{\gamma \delta}
$$


except that each of the two terms in (77) gives a unit contribution when $\alpha=\gamma=\beta=\delta$, while the matrix element $\Lambda_{\alpha \gamma \beta \delta}$ is still unity. However, the full sum in (71), including the standard basis, just adds an extra copy of precisely this case, so that (up to normalization) (77) are the matrix elements of $\Upsilon$ in the standard basis. These matrix elements are precisely those which define $\Pi$.

I believe that the mutually unbiased bases defined by Wootters and Fields for $d=2^{n}$ also form spherical designs, but have not shown it. Indeed, it may be that any set of mutually unbiased bases necessarily forms a spherical 2-design. The converse is true: for a set of $d(d+1)$ vectors in $C^{d}$ to generate a spherical 2-design in $C P_{d-1}$, it is necessary that they be a set of $d+1$ MUBs. (This follows from Theorem 44.9 in [44.)

These designs have an interesting relation to quantum error-correcting codes, and are also relevant in cryptography, where they serve to provide a finite ensemble with average-disturbance properties similar to those for the uniform ensemble. The informationdisturbance tradeoff is central to the power of quantum cryptography. The existence of such finite ensembles may serve in some cases to allow specification of key or proto-key material with a finite amount of information, while retaining the strong average-disturbance properties of states completely unknown to one without the key information. For example, these bases may serve to define the obvious $d(d+1)$-state generalization of the 6 -state protocol ( [45], [46]) on qubits.

\section{CONCLUSION}

We have defined and investigated properties of the information-disturbance frontier for quantum measurements on an ensemble of states on a finite dimensional Hilbert space, as a particular way of formalizing the intuitive notion that quantum mechanics often enforces

a tradeoff between gaining information and causing disturbance. General properties of the frontier, such as its convexity and monotonicity were established.

Specializing to important case of the uniform ensemble, representing a complete lack of knowledge about the initial state, we established further results concerning information and disturbance. For any measurement on this ensemble, we showed that a least-disturbing way of doing it causes the system to suffer a dynamics, conditional on each measurement result, described by a single Hellwig-Kraus operator. We also established that if we restrict ourselves to operations for which all Hellwig-Kraus operators are positive (so that they represent the square-root conditional dynamics for some measurement), a least-disturbing operation compatible with a given measurement, for any ensemble, is to do the squareroot dynamics for that measurement: fine-graining the measurement can never reduce the disturbance. However, we did not establish this for general conditional dynamics, leaving as an interesting open question whether there are non-uniform ensembles for which the least disturbing way of doing a particular measurement is for the apparatus to collect additional information beyond the measurement outcomes, and use it to aid in attempting to restore the initial state. Our main result establishes that this is not so for the uniform ensemble, since an optimal instrument for measuring it just implements the square-root conditional dynamics. This allows us to calculate, for any measurement, the minimal disturbance compatible with a measuring it on the uniform ensemble. This is only part of what is necessary to find the information-disturbance frontier, which involves an in general difficult maximization of 
accessible information subject to a disturbance constraint. We showed that the maximal information on the uniform ensemble may be obtained by unitarily covariant measurements and conditional dynamics. Thus, the overall action of the measurement dynamics on the state is just that of a "generalized depolarizing channel" family of operations depending on a single parameter $p$ which either do nothing to the state, or replace it with the maximally mixed state with probability $p$. It remains only to find the optimal measurement compatible with the generalized depolarizing channel as a function of that parameter $p$. Thus the the problem of determining the information disturbance frontier for the uniform ensemble is reduced from solving a parametric family of constrained maximization problems to solving a simpler parametric family of unconstrained ones.

\section{ACKNOWLEDGMENTS}

Some of the work reported here was carried out at the Center for Advanced Studies of the University of New Mexico, and appeared in my UNM doctoral dissertation [47]. This work was supported in part by Office of Naval Research Grant No. N00014-93-1-0116, National Science Foundation Grant No. PHY-9722614, the Institute for Scientific Interchange Foundation, Turin, Italy and Elsag, a Finmeccanica company, and the European Union project QAIP, IST-1999-11234. I thank Carlton M. Caves, Chris Fuchs, and Richard Jozsa for discussions, and an anonymous referee for valuable comments and improvements. The ideas herein originated from suggestions of Chris Fuchs that one should look for an "informationdisturbance uncertainty relation," and my interest in characterizing the projection postulate as somehow minimally disturbing, and were developed concurrently with Fuchs and Peres own development of the same formalism. Richard Jozsa suggested the role of the square root conditional dynamics.

\section{APPENDIX A: SINGLE-TERM CONDITIONAL OPERATIONS MINIMIZE UNIFORM ENTANGLEMENT DISTURBANCE}

Theorem 12 Let $\left\{F_{b}\right\}$ be a POVM and $\mathcal{F}_{b} \sim\left\{F_{b}^{1 / 2}\right\}$, and $\mathcal{A}=\sum_{b} \mathcal{A}_{b} \sim\left\{A_{b i}\right\}$, with $\sum_{i} A_{b i}^{\dagger} A_{b i}=F_{b}$, be trace-preserving operations. Then

$$
F_{e}(I / d, \mathcal{A}) \leq F_{e}\left(I / d, \mathcal{F}_{b}\right) .
$$

Proof: We decompose $\mathcal{A}_{b}$ into the composition of two operations: a trace-decreasing operation $\mathcal{G}_{b}$ defined by:

$$
\mathcal{F}_{b}(\rho)=F_{b}^{1 / 2} \rho F_{b}^{1 / 2}
$$

and an operation $\mathcal{B}_{b}$ (which is trace-preserving on the support of $F_{b}$ ) defined by:

$$
\mathcal{B}_{b}(\rho)=\sum_{i} B_{b i} \rho B_{b i}^{\dagger},
$$

where $B_{b i}=A_{b i} F_{b}^{-1 / 2}$. ( $F_{b i}$ may not be invertible; in this case, $F_{b i}^{-1 / 2}$ refers to the square root of the generalized inverse of $F_{b i}$. The generalized inverse is the inverse on $F_{b i}$ 's support (where 
it is invertible) extended (as a direct sum) by the zero operator on the orthocomplement of the support.) It is easily seen that $\mathcal{B}_{b}$ is trace-preserving on $F_{b}$ 's support $\left(\sum_{i} B_{i}^{\dagger} B_{i}=\Pi_{b}\right.$, where $\Pi_{b}$ is the projector onto the support of $F_{b}$ ), and that $\mathcal{B}_{b} \circ \mathcal{F}_{b}=\mathcal{A}_{b}$.

Then

$$
F_{e}(I / d, \mathcal{A})=\frac{1}{d^{2}} \sum_{b i}\left|\operatorname{tr} B_{b i} F_{b}^{1 / 2}\right|^{2} .
$$

By the Schwarz inequality,

$$
\left|\operatorname{tr} B_{b i} F_{b}^{1 / 2}\right|^{2} \equiv\left|\operatorname{tr} B_{b i} F_{b}^{1 / 4} F_{b}^{1 / 4}\right|^{2} \leq\left(\operatorname{tr} B_{b i} F_{b}^{1 / 2} B_{b i}^{\dagger}\right)\left(\operatorname{tr} F_{b}^{1 / 2}\right),
$$

so by the trace-preserving property for each $\mathcal{B}_{b}$,

$$
F_{e}(I / d, \mathcal{A}) \leq \frac{1}{d^{2}} \sum_{b}\left|\operatorname{tr} F_{b}^{1 / 2}\right|^{2}=F_{e}(I / d, \mathcal{G}) .
$$

This is just the entanglement fidelity for the uniform density operator when the operation $\mathcal{G}$ corresponding to the generalized Lüders' rule is used. Hence the generalized projection postulate minimizes disturbance to the entanglement of the uniform density operator.

\section{APPENDIX B: MORE ON ONE-TERM VERSUS MULTI-TERM CONDITIONAL OPERATIONS}

Here I consider some other approaches towards proving Theorem 5. These have so far proven unsuccessful except in the case in which all POVM elements commute. They are still of some interest in that they attempt to establish intermediate results stronger than Proposition 6.

In the one-term conditional dynamics case, we had:

$$
\left|\left\langle\psi\left|A_{b}\right| \psi\right\rangle\right|^{2} \leq\left|\left\langle\psi\left|F_{b}\right| \psi\right\rangle\right|^{2}
$$

In the multiple-term conditional dynamics case, we might hope to establish that

$$
\sum_{i}\left|\left\langle\psi\left|A_{b i}\right| \psi\right\rangle\right|^{2} \leq\left|\left\langle\psi\left|F_{b}\right| \psi\right\rangle\right|^{2}
$$

If $A_{b i}$ is assumed positive (as it is for the conditional dynamics which are minimallydisturbing to the uniform ensemble), this follows from Proposition 6, but we might try to establish B2 without that assumption. (There is no hope of estabilishing that $U_{b i}=I$, i.e. $A_{b i}$ positive, is minimally disturbing for an arbitary ensemble; it is obviously not true, for example, when the ensemble has all probability concentrated on one state $|\psi\rangle$.)

Defining $\mathcal{B}_{b}$ and $B_{i}$ as in Appendix A,

$$
\begin{aligned}
\left|\left\langle\psi\left|A_{b i}\right| \psi\right\rangle\right|^{2} & =\left|\left\langle\psi\left|B_{b i} F_{b}^{1 / 2}\right| \psi\right\rangle\right|^{2} \\
& =\left|\left\langle\psi\left|B_{b i} F_{b}^{1 / 4} F_{b}^{1 / 4}\right| \psi\right\rangle\right|^{2} .
\end{aligned}
$$

Applying the Schwarz inequality as before gives 


$$
\begin{aligned}
& \left|\left\langle\psi\left|B_{b i} F_{b}^{1 / 4} F_{b}^{1 / 4}\right| \psi\right\rangle\right|^{2} \\
\leq & \left\langle\psi\left|B_{b i} F_{b}^{1 / 2} B_{b i}^{\dagger}\right| \psi\right\rangle\left\langle\psi\left|F_{b}^{1 / 2}\right| \psi\right\rangle .
\end{aligned}
$$

If the inner product were a trace, as before, we would just cycle $B_{b i}^{\dagger}$ next to $B_{b i}$ and then sum on $i$ to get the identity, removing the $B$ 's entirely and establishing equation (B2). Unfortunately, we cannot do that here unless $B_{b i}$ commutes with $F_{b}$. Nor is it clear we can cycle one of the $F_{b}^{1 / 4}$ in $(\mathbb{B} 3)$ around to give $\left|\left\langle\psi\left|F_{b}^{1 / 4} B_{b i} F_{b}^{1 / 4}\right| \psi\right\rangle\right|^{2}$, which would have given rise to the desired ordering after the Schwarz inequality was applied. To proceed from B4 means we are trying to show that:

$$
\sum_{i}\left\langle\psi\left|B_{b i} F_{b}^{1 / 2} B_{b i}^{\dagger}\right| \psi\right\rangle \leq\left\langle\psi\left|F_{b}^{1 / 2}\right| \psi\right\rangle
$$

using the fact that $\sum_{i} B_{b i}^{\dagger} B_{b i}=I$. However, counterexamples to

$$
|\langle\psi|\mathcal{E}(G)| \psi\rangle| \leq|\langle\psi|G| \psi\rangle|
$$

for trace-preserving $\mathcal{E}$ and $0 \leq G \leq I$ are easily found. For example, let $G$ be proportional to a projector onto some state other than $|\psi\rangle$, and let $\mathcal{E}$ unitarily rotate that state back to $|\psi\rangle$. Since $\mathcal{B}_{b}$ may be an arbitrary trace-preserving operation, this means that the Schwarz inequality as applied to obtain equation (B4) is too loose for our purposes, and we must work with (B3), summed over $i$.

In fact, the counterexample to $(\bar{B} 6)$ given above also shows that even this will not work: there is no hope of establishing equation (B2), because it is equivalent to:

$$
\langle\psi|\mathcal{B F}(|\psi\rangle\langle\psi|)| \psi\rangle \leq\langle\psi|\mathcal{F}(|\psi\rangle\langle\psi|)| \psi\rangle
$$

Rather, we might try to show that

$$
\langle\psi|\mathcal{B F}(|\psi\rangle\langle\psi|)| \psi\rangle \leq \max _{\text {unitary } U}\left\langle\psi\left|U \mathcal{F}(|\psi\rangle\langle\psi|) U^{\dagger}\right| \psi\right\rangle
$$

where $\mathcal{B}$ is arbitrary and trace-preserving and $\mathcal{F} \sim\{F\}, I \geq F \geq 0$. 


\section{REFERENCES}

[1] C. H. Bennett and G. Brassard, in Proceedings of IEEE International Conference on Computers, Systems, and Signal Processing, Bangalore, India (IEEE Press, ADDRESS, 1985), pp. 175-179.

[2] C. H. Bennett, Physical Review Letters 68, 3121 (1992).

[3] J. Watrous, (2000), lANL arXiv.org e-print quant-ph/9901015.

[4] J. von Neumann, Tomsk. Univ. Rev. 1, 286 (1937).

[5] C. Cohen-Tannoudji, B. Diu, and F. Laloë, Quantum Mechanics (John Wiley and Sons, New York, 1977).

[6] G. Lüders, Annalen der Physik 8, 323 (1951).

[7] G. Ludwig, Foundations of Quantum Mechanics I (Springer, New York, 1983a), translation of Die Grundlagen der Quantenmechanik, Springer 1954.

[8] K. Kraus, States, Effects, and Operations (Springer-Verlag, Berlin, 1983).

[9] E. B. Davies, IEEE Transactions on Information Theory IT-24, 596 (1978).

[10] M. Ozawa, Reports on Mathematical Physics 18, 11 (1980).

[11] K. Hellwig and K. Kraus, Commun. Math. Phys. 16, 142 (1970).

[12] M.-D. Choi, Linear Algebra and Its Applications 10, 285 (1975).

[13] E. B. Davies and J. T. Lewis, Communications in Mathematical Physics 17, 239 (1970).

[14] Y. C. Eldar and G. D. Forney, (2000), to appear in IEEE Transactions on Information Theory.

[15] D. Bures, Transactions of the American Mathematical Society 135, 199 (1969).

[16] A. Uhlmann, Reports on Mathematical Physics 9, 273 (1976).

[17] R. Jozsa, Journal of Modern Optics 41(12), 2314 (1994).

[18] C. Fuchs and A. Peres, Physical Review A 53, 2038 (1995).

[19] B. W. Schumacher, Physical Review A 54, 2614 (1996).

[20] R. Jozsa, D. Robb, and W. K. Wootters, Physical Review A 49, 668 (1994).

[21] R. A. Horn and C. R. Johnson, Matrix Analysis (Cambridge University Press, Cambridge, 1985).

[22] T. Ando, Linear Algebra and its Applications 26, 203 (1979).

[23] E. Lieb, Advances in Mathematics 11, 267 (1973).

[24] R. Bhatia, Matrix analysis (Springer, Berlin, 1997).

[25] P. L. R. Ahlswede, (1999), lANL arXiv.org preprint quant-ph/9907081.

[26] A. Ashikmin, A. Barg, E. Knill, and S. Litsyn, IEEE Transactions on Information Theory 46, 778 (2000).

[27] K. R. W. Jones, Journal of Physics A 24, 121 (1991).

[28] W. K. Wootters, Foundations of Physics 20(11), 1365 (1990).

[29] E. B. Davies, Quantum Theory of Open Systems (Academic, London, 1976v).

[30] A. S. Holevo, Journal of Mathematical Physics 39, 1373 (1998).

[31] R. Werner, Physical Review A 58, 1827 (1996).

[32] P. Zanardi, Phys. Rev. A 58, 3484 (1998).

[33] M. Keyl and R. Werner, J. Math. Phys 40, 3283 (1999).

[34] W. F. Stinespring, Proc. Amer. Math. Soc. 6, 211 (1955).

[35] W. K. Wootters and B. D. Fields, Annals of Physics 191, 368 (1989).

[36] S. Wiesner, SIGACT News 15, 78 (1983).

[37] I. D. Ivanović, J. Math. Phys. A: Math. Gen. 14, 3241 (1981). 
[38] F. J. MacWilliams and N. J. A. Sloane, The Theory of Error-Correcting Codes (NorthHolland, Amsterdam, 1977).

[39] R. Lidl and H. Niederreiter, Introduction to finite fields and their applications (Cambridge University Press, Cambridge, 1986).

[40] A. R. Calderbank, P. J. Cameron, W. M. Kantor, and J. J. Seidel, Proc. London Math. Soc. 3, 436 (1997).

[41] P. Delsarte, J. M. Goethals, and J. J. Seidel, Phillips Res. Repts. 30, 91 (1975).

[42] E. Rains, LANL e-print quant-ph/9704042 (1997).

[43] M. Grassl, M. Rötteler, and T. Beth, Physical Review A 58, 1833 (1998).

[44] S. Hoggar, in CRC Handbook of Combinatorial Designs (CRC Press, Toronto, 1996), p. 461.

[45] D. Bruß, A. Ekert, and C. Macchiavello, LANL e-print quant-ph/9712019 (1997).

[46] D. Bruß, Physical Review Letters 81, 3018 (1998).

[47] H. Barnum, (1998), UNM Doctoral Dissertation. 